Physics

Physics Research Publications

Chandra view of the unidentified $\mathrm{TeV}$ gamma-ray source HESS J1804-216

W. Cui A. Konopelko 


\title{
CHANDRA VIEW OF THE UNIDENTIFIED TeV GAMMA-RAY SOURCE HESS J1804-216
}

\author{
Wei Cui and Alexander Konopelko \\ Department of Physics, Purdue University, 1396 Physics Building, West Lafayette, IN 47907 \\ Received 2006 September 8; accepted 2006 October 23; published 2006 November 9
}

\begin{abstract}
We present high-resolution X-ray images taken with the Chandra X-Ray Observatory of the field that contains the unidentified TeV gamma-ray source HESS J1804-216. A total of 11 discrete sources were detected with a posteriori significance of $>5 \sigma$ over the entire field of view. Among them, only one, designated as CXOU J180351.4-213707, is significantly extended. The source is about $40^{\prime \prime}$ away from the radio pulsar PSR J1803-2137, which was the target of the Chandra observation but was not detected in X-rays. A natural question is whether the two sources are physically related. While it is conceivable that CXOU J180351.4-213707 could be associated with a previously unknown supernova remnant (SNR), in which the pulsar was born, it seems equally plausible that it might be a pulsar wind nebula (PWN) that is powered by a different pulsar whose emission is beamed away from us. In either case, we argue that CXOU J180351.4-213707 is likely the X-ray counterpart of HESS J1804-216, based on the fact that the Galactic TeV gamma-ray sources are predominantly SNRs or PWNe. The X-ray spectrum of the source can be well fitted with a power law, although the model is not well constrained due to large statistical uncertainties. The spectrum seems to be very hard, with a best-fit photon index of about 1.2. Under the assumption that CXOU J180351.4-213707 is the X-ray counterpart of HESS J1804-216, we attempted to model the X-ray and TeV emission as synchrotron and inverse Compton scattered radiation from relativistic electrons. We briefly discuss the results.
\end{abstract}

Subject headings: acceleration of particles — gamma rays: theory — pulsars: individual (PSR J1803-2137) — radiation mechanisms: nonthermal — supernova remnants - X-rays: general

\section{INTRODUCTION}

One of the most exciting recent advances in high-energy astrophysics is the detection of various classes of sources at $\mathrm{TeV}$ energies with ground-based gamma-ray facilities (for recent reviews, see Weekes 2006 and Cui 2006). The established TeV gamma-ray emitters now include blazars, radio galaxies, shelltype supernova remnants (SNRs), pulsar wind nebulae (PWNe), microquasars, and Be binaries, all of which have traditionally been standard targets for X-ray/soft gamma-ray observations. Arguably the most significant discovery, however, is the presence of a population of unidentified $\mathrm{TeV}$ gamma-ray sources. These sources are probably Galactic in origin, given their concentration around the Galactic plane (although one must take into account a strong observational bias toward the Galactic plane). Some of them have plausible counterparts at longer wavelengths (Aharonian et al. 2005a, 2006a), based mostly on positional coincidence, but others have none at all (Mukherjee et al. 2003; Butt et al. 2003; Aharonian et al. 2005a, 2006a).

A number of proposals have been made on the nature of the unidentified $\mathrm{TeV}$ gamma-ray sources. The sources might be associated with old shell-type SNRs (Yamazaki et al. 2006). Such systems could have a very high ratio of $\mathrm{TeV}$ to X-ray (or radio) fluxes, which makes them difficult to detect at low energies. For instance, HESS J1813-178 was initially "dark" (Aharonian et al. 2005a) but was subsequently identified with a shell-type SNR (G12.8-0.0; Brogan et al. 2005). On the other hand, HESS J1825-137 is now positively associated with a PWN (G18.0-0.7; Aharonian et al. 2006c). Therefore, both SNRs and PWNe are viable candidates. It is worth noting that the plausible counterparts of unidentified $\mathrm{TeV}$ gamma-ray sources are mostly shell-type SNRs or PWNe (Aharonian et al. 2005a, 2006a). Other possibilities also exist. For instance, HESS J1303-631 is postulated as the remnant of a gammaray burst that occurred some tens of thousands of years ago in our Galaxy (Atoyan et al. 2006). TeV J2032+4130 might be associated with shocks produced by colliding winds of massive stars in the dense Cyg OB2 association (Butt et al. 2003).

In this Letter, we report the detection of extended X-ray emission near the radio pulsar PSR J1803-2137. The pulsar has been suggested as a possible counterpart of HESS J1804-216, one of the brightest unidentified $\mathrm{TeV}$ gamma-ray sources (Aharonian et al. 2005a, 2006a). While our results cannot definitively establish a physical connection between the extended emission and the pulsar, they have provided evidence to suggest that the former may be the X-ray counterpart of HESS J1804-216.

\section{DATA ANALYSIS AND RESULTS}

The data for this work were derived from an archival Chandra observation of PSR J1803-2137 (ObsID 5590). The data were taken with the ACIS detector, with CCDs I2-3 and S0-3 being read out. The aim point is on the S3 chip (with the default $Y$ offset $\Delta Y=-20^{\prime \prime}$ ). The total on-source time is about $30 \mathrm{ks}$. The data were reduced with the standard CIAO analysis package (ver. 3.3), along with CALDB 3.2.0. We followed the CIAO Science Threads ${ }^{1}$ in preparing the data (including bad pixel removal and data filtering) and constructing images and spectra. Little data were filtered out in this case, so the effective exposure time is also about $30 \mathrm{ks}$. We found that the Level 2 event file (with a processing version of 7.6.7) from the archive had already incorporated the most updated calibrations, so we used it as a starting point for subsequent imaging and spectral analysis.

\subsection{Imaging Analysis}

We made an X-ray image of the field in the $0.5-10 \mathrm{keV}$ band and carried out a search for discrete sources. The CIAO tool celldetect was used. It is based on the sliding-cell algorithm, but the detection cell is allowed to vary in size to match the

\footnotetext{
${ }^{1}$ See http://asc.harvard.edu/ciao/threads/index.html.
} 
TABLE 1

Detected X-Ray Sources

\begin{tabular}{ccccc}
\hline $\begin{array}{c}\text { Name } \\
(\text { CXOU })\end{array}$ & \multicolumn{1}{c}{$\begin{array}{c}\text { R.A. } \\
(\mathrm{J} 2000)\end{array}$} & $\begin{array}{c}\text { Decl. } \\
(\mathrm{J} 2000)\end{array}$ & $\begin{array}{c}\text { Count Rate } \\
\left(10^{-3} \text { counts s }^{-1}\right)\end{array}$ \\
\hline $\mathrm{J} 180341.5-214034 \ldots \ldots$ & $180341.54(2)$ & $-214034.8(2)$ & $1.27 \pm 0.22$ \\
$\mathrm{~J} 180345.3-213038 \ldots \ldots$ & $180345.34(1)$ & $-213038.7(2)$ & $2.42 \pm 0.37$ \\
$\mathrm{~J} 180349.1-212317 \ldots \ldots$ & $180349.19(3)$ & $-212317.2(4)$ & $4.34 \pm 0.79$ \\
$\mathrm{~J} 180349.3-214135 \ldots \ldots$ & $180349.35(2)$ & $-214135.8(2)$ & $1.54 \pm 0.26$ \\
$\mathrm{~J} 180350.9-213837 \ldots \ldots$ & $180350.94(1)$ & $-213837.8(1)$ & $1.48 \pm 0.25$ \\
$\mathrm{~J} 180351.4-213707 \ldots \ldots$ & $180351.411(4)$ & $-213707.37(5)$ & $4.25 \pm 0.32$ \\
$\mathrm{~J} 180355.0-213937 \ldots \ldots$ & $180355.00(1)$ & $-213937.4(1)$ & $1.12 \pm 0.17$ \\
$\mathrm{~J} 180400.7-214251 \ldots \ldots$ & $180400.76(2)$ & $-214251.5(2)$ & $2.41 \pm 0.32$ \\
$\mathrm{~J} 180401.2-213153 \ldots \ldots$ & $180401.217(5)$ & $-213153.48(7)$ & $6.23 \pm 0.53$ \\
$\mathrm{~J} 180404.2-213709 \ldots \ldots$ & $180404.27(1)$ & $-213709.5(1)$ & $0.97 \pm 0.19$ \\
$\mathrm{~J} 180432.4-214009 \ldots \ldots$ & $180432.47(2)$ & $-214009.8(3)$ & $4.58 \pm 0.45$ \\
\hline
\end{tabular}

Note. - Units of right ascension are hours, minutes, and seconds, and units of declination are degrees, arcminutes, and arcseconds. The coordinates and count rates shown are based on the output of celldetect, except for CXOU J180351.4-213707 (see text). The numbers in parentheses indicate uncertainty in the last digit. Note that only statistical uncertainties are shown.

local point-spread function (PSF). The key parameters are the signal-to-noise ratio threshold and the size of the detection cell, which are, by default, set at 3 and $80 \%$ of the encircled energy of the PSF, respectively. We also left all other parameters at the default values. The default settings have been shown to be effective against spurious detections. ${ }^{2}$ We assessed the statistical significance of each detection using equation (17) in $\mathrm{Li} \& \mathrm{Ma}$ (1983), based on the output of celldetect (namely, the sizes of the source and background regions and the total counts in the two regions). Table 1 shows all detections with significance $\gtrsim 5 \sigma$. Seven of the sources are on the S3 chip, two on the S2 chip, two on the S1 chip, and one on the I3 chip.

A few remarks on the results are necessary. First of all, only statistical uncertainties are shown for the best-fit positions of the sources. It is known that the systematic uncertainty on the absolute position of a source can be much larger, about 0.6 in radius of the $90 \%$ error circle and $00^{\prime \prime} 8$ in radius of the $99 \%$ error circle on

${ }^{2}$ See http://asc.harvard.edu/ciao/download/doc/detect_manual/cell_false.html.

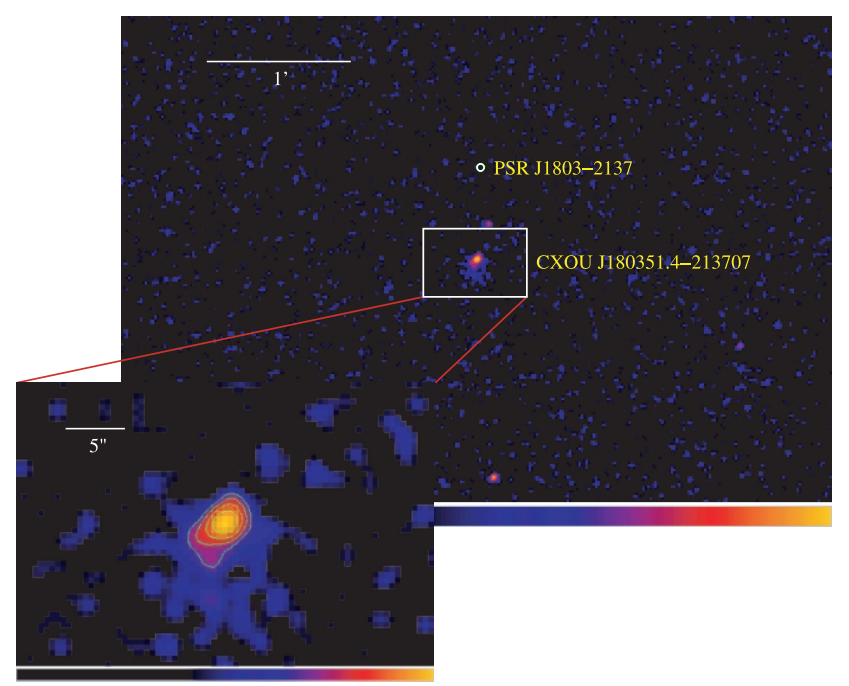

FIG. 1.-X-ray view of PSR J1803-2137 and its surroundings. The image was made in the $0.5-10 \mathrm{keV}$ band. It has been smoothed and is shown on a logarithmic scale. The pulsar is not detected; its radio position is indicated by an open circle. On the other hand, the presence of CXOU J180351.4-213707 is apparent. The inset shows an expanded view of the source, with contours (at the levels of $0.50,0.87,1.52$, and 2.64 counts) overlaid to show its extension. average (but larger for sources more than $3^{\prime}$ from the aim point). ${ }^{3}$ Second, for extended sources, celldetect only determines the coordinates of the centroid. Third, the count rates shown are also from celldetect, except for CXOU J180351.4-213707, which celldetect failed to adequately characterize, due to its extended nature. To accurately extract source counts from CXOU J180351.4-213707, we used a circular source region that was centered on it with a radius of 10 pixels (or about $5^{\prime \prime}$ ) and a concentric background annulus with an inner radius of 10 pixels and an outer radius of 25 pixels (chosen to avoid a possible source that is below our detection threshold; see Fig. 1). The true count rate of the source is shown in Table 1.

Among the sources that we detected, CXOU J180351.4213707 is the only one that is significantly extended. Figure 1 shows an expanded view of the source, along with a broad view of PSR J1803-2137 and its surroundings. The image has been smoothed (in ds9) with a Gaussian kernel that is 3 pixels in radius. CXOU J180351.4-213707 is seen to extend roughly 7 " along both right ascension and declination. It is about $40^{\prime \prime}$ away from PSR J1803-2137. No X-ray emission from the latter is detected. To be more quantitative, we extracted counts from a circular source region that is 5 pixels in radius and centered at the position of the pulsar and from a concentric background annulus with an inner radius of 10 pixels and outer radius of 40 pixels. The resulting net count rate is $(-5 \pm 9) \times 10^{-5}$ counts $\mathrm{s}^{-1}$.

We searched the SIMBAD and NED databases for plausible counterparts of the detected sources. Within a 30" search radius, we found only one candidate, 1WGA J1803.6-2140, which is about $12^{\prime \prime}$ away from CXOU J180341.5-214034. In addition, CXOU J180432.4-214009 appears to be the same source as Suzaku J1804-2140 (Bamba et al. 2006), which was suggested as a plausible X-ray counterpart of HESS J1804-216, although the source is not obviously extended in our case. We should note that this source falls on the $\mathrm{I} 3$ chip, which is quite far from the aim point. No X-ray emission was detected at the position of Suzaku J1804-2142 (Bamba et al. 2006), implying that it is either a transient or highly variable source.

\subsection{Spectral Analysis}

We used the CIAO script specextract to construct the Xray spectrum of CXOU J180351.4-213707. Here we adopted the same source region as before but used a much larger back-

${ }^{3}$ See http://asc.harvard.edu/cal/ASPECT/celmon/. 


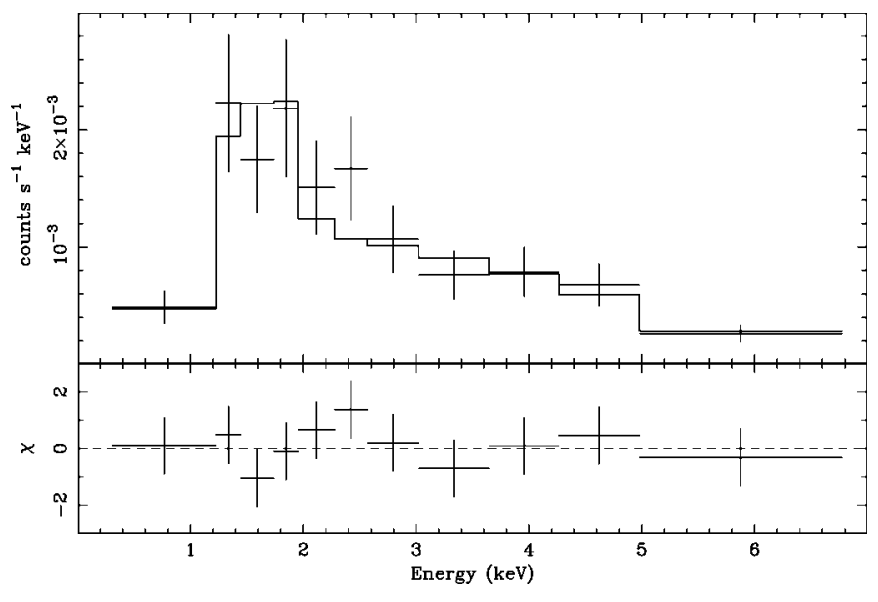

FIG. 2.-X-ray spectrum of CXOU J180351.4-213707. The solid histogram in the top panel shows the best fit to the data with an absorbed power-law model. The residuals are shown in the bottom panel.

ground annulus, the outer radius of which is 170 pixels. We excluded a circular region (of 9 pixel radius) that is centered on the possible source to the northwest of CXOU J180351.4213707 , just to be on the cautious side. The script produced both the overall and background spectra, as well as the corresponding redistribution matrix file and auxiliary response file that are needed for subsequent spectral modeling.

For spectral analysis, we excluded data points below $0.3 \mathrm{keV}$ and above $10 \mathrm{keV}$ and then rebinned the raw spectrum so that there are at least 15 counts in each bin. We carried out spectral modeling in XSPEC 11.3.2 (Arnaud 1996). The backgroundsubtracted spectrum is shown in Figure 2. It can be well fitted with a simple absorbed power-law model (wabs*powerlaw in XSPEC). The best-fit model and residuals are also shown in Figure 2. The reduced $\chi^{2}$ of the fit is 0.57 for 8 degrees of freedom. The derived parameters are hydrogen column density $N_{\mathrm{H}}=0.8_{-0.3}^{+0.6} \times 10^{22} \mathrm{~cm}^{-2}$, photon index $\Gamma=1.2_{-0.4}^{+0.5}$, and normalization $K=1.1_{-0.5}^{+1.2} \times 10^{-5}$ photons $\mathrm{cm}^{-2} \mathrm{~s}^{-1} \mathrm{keV}^{-1}$ at $1 \mathrm{keV}$. The errors shown represent $90 \%$ confidence limits. Although the model is not well constrained, due to large statistical uncertainties, CXOU J180351.4-213707 seems to be a very hard X-ray source. The spatially averaged (absorbed) flux of the source is $1.0_{-0.9}^{+29.3} \times 10^{-13} \mathrm{ergs} \mathrm{cm}^{-2} \mathrm{~s}^{-1}$ (in the $0.3-10 \mathrm{keV}$ band).

\section{DISCUSSION}

The Chandra observation has revealed the presence of 11 discrete X-ray sources in the general vicinity of HESS J1804216. Figure 3 shows the positions of these sources in Galactic coordinates, as overlaid over the $\mathrm{TeV}$ gamma-ray image of the field (Aharonian et al. 2006a). While it is difficult to be certain as to which is the counterpart of HESS J1804-216, we argue that CXOU J180351.4-213707 is most probable. It is the only significantly extended source detected. It is conceivable that the source might be a previously unknown SNR that is associated with PSR J1803-2137. If so, the spatial offset between the two could be attributed to the proper motion of the pulsar. At the distance of the pulsar ( 4 kpc; Taylor \& Cordes 1993), the separation between the two is only about $0.78 \mathrm{pc}$. Assuming the pulsar is born at the "center" of CXOU J180351.4-213707, it would only require a transverse speed of $\$ 50 \mathrm{~km} \mathrm{~s}^{-1}$ for it to reach the current position in $\sim 16,000$ years, which is its characteristic spin-down age $(\equiv P / 2 \dot{P}$; Clifton \& Lyne 1986). This

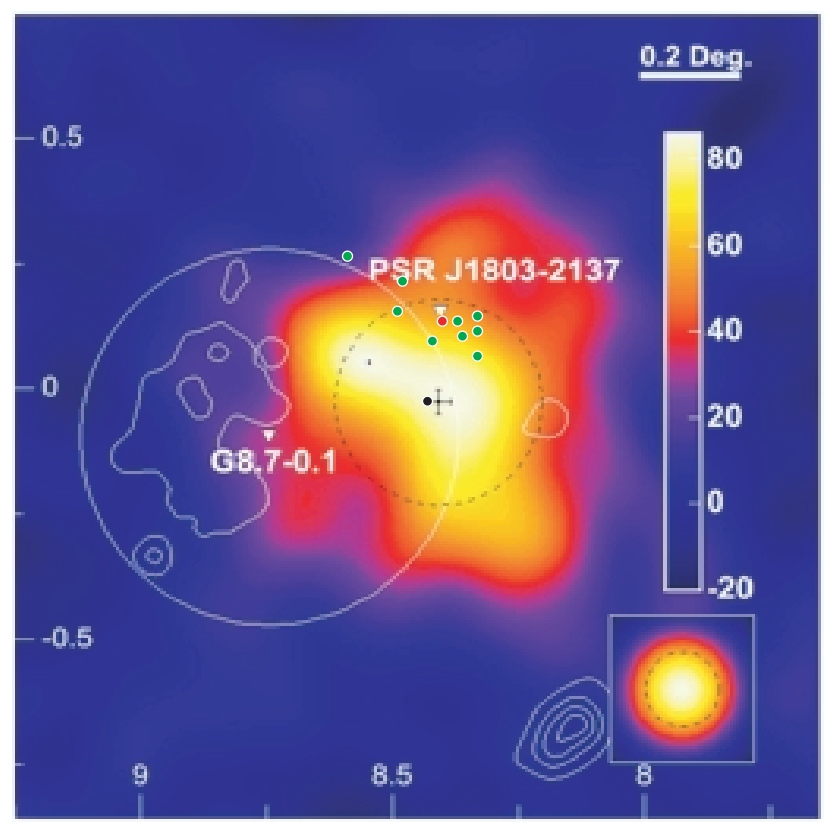

FIG. 3.-X-ray sources in the vicinity of HESS J1804-216. The TeV gamma-ray image was adapted from Aharonian et al. (2006a). The dashed circle is meant to show the average extent of the gamma-ray emission region. The positions of the detected X-ray sources are indicated by filled circles. Highlighted are CXOU J180351.4-213707 (in red), which might be the Xray counterpart of HESS J1804-216, and CXOU J180432.4-214009 (in black), which is likely the same source as Suzaku J1804-2140. The ROSAT contours of G8.7-0.1 (Finley \& Ögelman 1994) are also overlaid. The white circle shows the extent of the $20 \mathrm{~cm}$ emission as measured with the Very Large Array.

would be easily achievable. However, the measured size of CXOU J180351.4-213707 is only about 0.14 pc (for an angular size of $\sim 7^{\prime \prime}$ ), which seems to be much too small for an SNR that is over $10^{4}$ yr old.

On the other hand, Chandra might have revealed only the brightest part of the hypothesized SNR, and its true size might be much larger. In fact, CXOU J180351.4-213707 might only represent a bright spot of X-ray emission of such an SNR. This scenario can be tested with a much deeper exposure of the field with Chandra or XMM-Newton in the future. It should be noted that PSR J1803-2137 was initially thought to be associated with the SNR G8.7-0.1 (Kassim \& Weiler 1990; see also Fig. 3), based on similar estimated distances and ages between the two. However, it was subsequently realized that an unusually large transverse velocity $\left(\sim 1700 \mathrm{~km} \mathrm{~s}^{-1}\right)$ would be required for the pulsar to reach the current position if it was born at the center of G8.7-0.1. This, along with a smaller revised distance to the pulsar and the lack of evidence for such a large transverse velocity, led to a disassociation between the two systems (Frail et al. 1994; see, however, Finley \& Ögelman 1994). There would be no similar issues with the scenario that we are postulating here, because the pulsar might be even closer to the dynamical center of the hypothesized SNR than to CXOU J180351.4213707 (about $0.78 \mathrm{pc}$, at the distance of PSR J1803-2137).

Alternatively, CXOU J180351.4-213707 might have no physical connection with PSR J1803-2137. Instead, it could be a PWN that is powered by a different pulsar whose emission is beamed away from us. Again, the true size of such a PWN could be much larger than what has been measured. For instance, CXOU J180351.4-213707 might be the bright compact core of the PWN. Such a core is known to exist in several PWNe. As 
an example, a torus-like compact X-ray-emitting region was seen around the Vela pulsar with Chandra (Helfand et al. 2001) inside a much larger nebula (Markwardt \& Ögelman 1995; Aharonian et al. 2006b). Also, a small extended X-ray feature was detected around PSR J1826-1334 with XMM-Newton, as part of a more extended nebula (Gaensler et al. 2003), whose asymmetric profile is the key to establishing a physical connection between this PWN and HESS J1825-137 (Aharonian et al. 2005b). In this case, the X-ray emission is much less extended than the gammaray emission, which might be attributable to the difference in the cooling times of the X-ray- and gamma-ray-emitting electrons (Aharonian et al. 2005b). The same might also be true for CXOU J180351.4-213707, if it is the X-ray counterpart of HESS J1804-216. Moreover, PSR J1826-1334 is offset from the gamma-ray peak of HESS J1825-137, as would be the case for CXOU J180351.4-213707/HESS J1804-216 if the PWN scenario holds.

Assuming that CXOU J180351.4-213707 is the X-ray counterpart of HESS J1804-216, we proceeded to assemble the spectral energy distribution (SED) of the system. Figure 4 shows the results. Since the X-ray-emitting region could be much larger, as already discussed, the measured X-ray fluxes should only be taken as lower limits. We attempted to model the SED in a leptonic scenario, in which the X-ray emission is assumed to originate from the synchrotron radiation from relativistic electrons in the region and the $\mathrm{TeV}$ gamma-ray emission from inverse Compton (IC) scattering of ambient photons by the same electrons. The spectral distribution of the electrons is assumed to be of the form $Q_{e} \propto \gamma^{-s} e^{-\gamma / \gamma_{\max }}$. We found a fairly good fit to the X-ray SED with $s=0.4$ and $\gamma_{\max } \simeq 10^{8}$, for a spherical emitting region of radius $R=10^{17} \mathrm{~cm}$ and magnetic field $B=3 \mu \mathrm{G}$, using the methodology described by Mastichiadis (1996). The synchrotron self-Compton emission from such an electron spectrum peaks at frequencies well above $10^{28} \mathrm{~Hz}$. The position of the IC peak remains essentially unchanged with the addition of the cosmic microwave background $(\mathrm{CMB})$ photons $\left(U_{\mathrm{CMB}}=\right.$ $0.33 \mathrm{eV} \mathrm{cm}^{-3}$ ), as shown in Figure 4, in conflict with the measured gamma-ray spectrum. Varying model parameters does not fundamentally improve the situation.

On the other hand, we could find a reasonable fit to the $\mathrm{TeV}$ gamma-ray spectrum (e.g., with $s \simeq 1.5, \gamma_{\max } \simeq 10^{7}$, and $B \simeq 3 \mu \mathrm{G})$, as also shown in Figure 4. However, the required electron spectrum deviates significantly from that needed to explain the X-ray emission. This perhaps argues for a multizone scenario. Intriguingly, in this case, an extrapolation of the IC

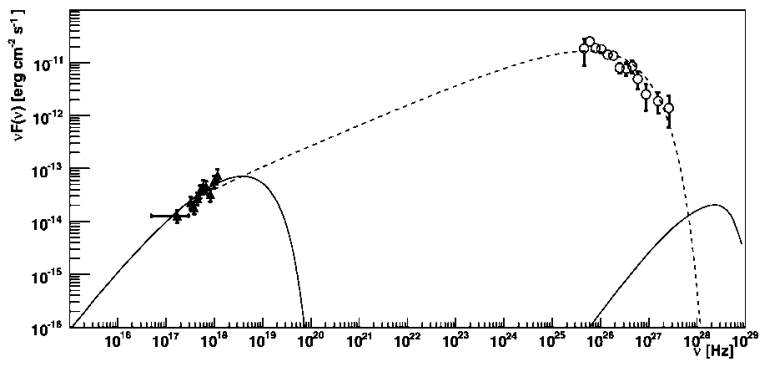

FIG. 4.-Combined spectral energy distribution of CXOU J180351.4213707 (filled triangles) and HESS J1804-216 (open circles). The solid curve shows representative results from leptonic calculations that aim at fitting the Xray data, while the dashed curve shows those that fit the gamma-ray data (see text).

spectrum comes very close to the X-ray measurements (see Fig. 4). Of course, one should always keep in mind the possibility that, e.g., CXOU J180351.4-213707 might be the compact core of a much extended PWN and that the true X-ray flux of the PWN might thus be much higher. More sophisticated modeling is beyond the scope of this work.

We conclude by noting the lack of X-ray emission from PSR J1803-2137. It is a bit surprising that this Vela-like pulsar could not be detected in a $30 \mathrm{ks}$ ACIS/Chandra observation. Given the measured $P(=133 \mathrm{~ms})$ and $\dot{P}\left(=1.34 \times 10^{-13} \mathrm{~s} \mathrm{~s}^{-1}\right)$, the spindown power of the pulsar $\left[\equiv-4 \pi^{2} I\left(\dot{P} / P^{3}\right)\right.$, where $I$ is the moment of inertia of the neutron star] is about $2.25 \times 10^{36} \mathrm{ergs} \mathrm{s}^{-1}$. Becker $\&$ Trümper (1997) showed that rotation-powered pulsars typically radiate away $0.1 \%$ of the spin-down power in the ROSAT band (0.1-2.4 keV). Therefore, we would expect a $0.1-2.4 \mathrm{keV} \mathrm{lu}-$ minosity of $2.25 \times 10^{33} \mathrm{ergs} \mathrm{s}^{-1}$ for PSR J1803-2137, or a flux of $1.2 \times 10^{-12} \mathrm{ergs} \mathrm{cm}^{-2} \mathrm{~s}^{-1}$, which is several orders of magnitude higher than the Chandra ( $1 \sigma$ ) upper limit of $1.7 \times 10^{-15}$ ergs $\mathrm{cm}^{-2} \mathrm{~s}^{-1}$ for the total emission.

We wish to thank the anonymous referee for his/her very useful comments. This research has made use of the NASA/ IPAC Extragalactic Database (NED), which is operated by the Jet Propulsion Laboratory, California Institute of Technology, under contract with the National Aeronautics and Space Administration, and of the SIMBAD Database. We gratefully acknowledge financial support from the Department of Energy.

\section{REFERENCES}

Aharonian, F., et al. 2005a, Science, 307, 1938

$$
\text { 2005b, A\&A, 442, L25 }
$$

2006a, ApJ, 636, 777

2006b, A\&A, 448, L43

$2006 \mathrm{c}, \mathrm{A} \& \mathrm{~A}$, in press

Arnaud, K. A. 1996, in ASP Conf. Ser. 101, Astronomical Data Analysis Software and Systems V, ed. G. Jacoby \& J. Barnes (San Francisco: ASP), 17

Atoyan, A., Buckley, J., \& Krawczynski, H. 2006, ApJ, 642, L153

Bamba, A., et al. 2006, PASJ, in press (astro-ph/0608310)

Becker, W., \& Trümper, J. 1997, A\&A, 326, 682

Brogan, C. L., Gaensler, B. M., Gelfand, J. D., Lazendic, J. S., Lazio, T. J. W., Kassim, N. E., \& McClure-Griffiths, N. M. 2005, ApJ, 629, L105

Butt, Y. M., et al. 2003, ApJ, 597, 494

Clifton, T. R., \& Lyne, A. G. 1986, Nature, 320, 43

Cui, W. 2006, in Frontier Objects in Astrophysics and Particle Physics, ed. F. Giovannelli \& G. Mannocchi (Bologna: Editrice Compositori), in press (astro-ph/0608042)
Finley, J. P., \& Ögelman, H. 1994, ApJ, 434, L25

Frail, D. A., Kassim, N. E., \& Weiler, K. W. 1994, AJ, 107, 1120

Gaensler, B. M., \& Schulz, N. S., Kaspi, V. M., Pivovaroff, M. J., \& Becker, W. E. 2003, ApJ, 588, 441

Helfand, D. J., Gotthelf, E. V., \& Halpern, J. P. 2001, ApJ, 556, 380

Kassim, N. E., \& Weiler, K. W. 1990, Nature, 343, 146

Li, T. P., \& Ma, Y. Q. 1983, ApJ, 272, 317

Markwardt, C. B., \& Ögelman, H. 1995, Nature, 375, 40

Mastichiadis, A. 1996, A\&A, 305, L53

Mukherjee, R., Halpern, J. P., Gotthelf, E. V., Eracleous, M., \& Mirabal, N. 2003, ApJ, 589, 487

Taylor, J. H., \& Cordes, J. M. 1993, ApJ, 411, 674

Weekes, T. C. 2006, in Energy Budget in the High Energy Universe, in press (astro-ph/0606130)

Yamazaki, R., Kohri, K., Bamba, A., Yoshida, T., Tsuribe, T., \& Takahara, F. 2006, MNRAS, 371, 1975 